\title{
ANCIENT SOLUTIONS FOR ANDREWS' HYPERSURFACE FLOW
}

\author{
PENG LU AND JIURU ZHOU
}

\begin{abstract}
We construct the ancient solutions of the hypersurface flows in Euclidean spaces studied by B. Andrews in 1994. As time $t \rightarrow 0^{-}$the solutions collapse to a round point where 0 is the singular time. But as $t \rightarrow-\infty$ the solutions become more and more oval. Near the center the appropriately-rescaled pointed Cheeger-Gromov limits are round cylinder solutions $S^{J} \times \mathbb{R}^{n-J}, 1 \leq J \leq n-1$. These results are the analog of the corresponding results in $\operatorname{Ricci}$ flow $(J=n-1)$ and mean curvature flow.
\end{abstract}

Keywords. Andrews' hypersurface flow, ancient solutions, asymptotic limits

MSC (2010). 53C44, 35K55, 58J35

\section{INTRODUCTION}

Ancient solutions are studied in various geometric flows including Ricci flow ([Pe02], [DHS12]), curve shortening flow ([DHS10]), and mean curvature flow (Wa11, Wh03, [HH16]) because of their close relation with the singularity analysis. In this article we use Perelman's idea in the construction of ancient oval solutions for Ricci flow and some ideas from [HH16] to construct ancient oval solutions for the Andrews' flow (An94]). Note that for mean curvature flow such a construction is done in the nice work of Haslhofer and Hershkovits ([HH16]). The uniqueness of the oval solutions for mean curvature flow is proved recently by Angenent, P. Daskalopoulos, and and N. Sesum ([ADS18]).

Now we recall the definition of Andrews' flow. Let $M^{n}$ be a smooth manifold of dimension $n \geq 2$ without boundary. For a smooth family of immersions $\varphi$ : $M \times[0, T) \rightarrow \mathbb{R}^{n+1}$ we denote hypersurface $\varphi(M \times\{t\})$ in $\mathbb{R}^{n+1}$ by $\sum_{t}^{n}$ and denote the unit normal vector of $\Sigma_{t}$ at $\varphi(p, t)$ by $\nu(\varphi(p, t), t)$ (chosen to be outward-pointing if the hypersurface is convex). We denote the principal curvatures $\Sigma_{t}^{n}$ at point $\varphi(p, t)$ by $\lambda_{t, 1}(\varphi(p, t)) \leq \cdots \leq \lambda_{t, n}(\varphi(p, t))$. Recall that a hypersurface is strictly convex if its principal curvatures are positive.

Define the cone $\Gamma_{+}^{n}=\left\{\left(\lambda_{1}, \cdots, \lambda_{n}\right) \in \mathbb{R}^{n}, \forall i, \lambda_{i}>0\right\}$. Let $f$ be a smooth positive function on $\Gamma_{+}^{n}$. Throughout this article we assume that $f$ is symmetric, homogeneous of degree one, and $\frac{\partial f}{\partial \lambda_{i}}>0$ for each $i$. We further make the following assumption for $f$ ( $\mathrm{An94}$, , An07]).

Assumption A. Let function $f^{*}$ be defined by $f^{*}\left(\lambda_{1}, \cdots, \lambda_{n}\right)=-f\left(\lambda_{1}^{-1}, \cdots, \lambda_{n}^{-1}\right)$. Function $f$ is assumed to satisfy one of the following assumption

(A1) $n=2$, or

(A2) $f$ is convex, or

(A3) $f$ is concave on $\Gamma_{+}^{n}$ and $f$ approaches zero on the boundary of $\Gamma_{+}^{n}$, or

(A4) both $f$ and $f^{*}$ are concave $\Gamma_{+}^{n}$.

Date: revised December 13, 2018. 
We consider the following equation of $\varphi$

$$
\frac{\partial}{\partial t} \varphi(p, t)=-f\left(\lambda_{t, 1}(\varphi(p, t)), \cdots, \lambda_{t, n}(\varphi(p, t))\right) \cdot \nu(\varphi(p, t), t) .
$$

We call the flow (1.1) the Andrews' flow. The solutions of Andrews' flow are necessarily strictly convex hypersurfaces. Below we use $S^{n}$ to denote the unit sphere and $S^{n}(R) \subset \mathbb{R}^{n+1}$ to denote the round sphere of radius $R$ centered at the origin.

Theorem 1.1. Fixing a $J \in\{1,2, \cdots, n-1\}$, we assume that the Condition $B_{J}$ holds for function $f$ (see \$2.2 for the definition), then there exists a non-spherical $O_{J+1} \times O_{n-J}$-rotationally symmetric ancient solution $\varphi_{J}: S^{n} \times(-\infty, 0) \rightarrow \mathbb{R}^{n+1}$ to Andrews' flow (1.1) which has strictly convex time slices $\hat{\Sigma}_{J, \infty, t}$ and which develops a singularity at time 0 . As $t \rightarrow 0$ - the solutions collapse to a round point.

Proposition 1.2. Assume $f$ in (1.1) satisfies Assumption E (see §3 for the definition). Then the solutions $\varphi_{J}$ constructed in Theorem 1.1 have the following backwards asymptotics: for $K \rightarrow \infty$ the parabolically-rescaled flows $K^{-1} \hat{\Sigma}_{J, \infty, K^{2} t}$ sub-converge to the round shrinking cylinder $S^{J}(\sqrt{2 J|t|}) \times \mathbb{R}^{n-J}$ for $t \leq-1$.

Remark 1.3. Corresponding to the choice of $J=0$ and for mean curvature flow Bourni, Langford and Tinglia construct compact convex and collapsing ancient solutions that lie in a slab with $O(1) \times O(n)$-symmetry (BLT17], see also [HIMW] and Wa11]). They further study the uniqueness of such solutions.

Acknowledgement. The authors would like to thank Mat Langford for bringing our attention to [BL16] and [BLT17]. J.R.Z. would like to thank China Scholarship Council for providing a fellowship as a visiting scholar at University of Oregon. P.L. is partially supported by Simons Foundation through Collaboration Grant 229727 and 581101. J.R.Z. is partially supported by a PRC grant NSFC 11426195.

\section{COMPARISON PRINCIPLE FOR ANDREWS' FLOW}

It is well-known that mean curvature flow satisfies a powerful comparison priciple ([Ec04]). We will need a comparison principle for Andrews' flow ([An94, Proof of Theorem 6.2] and [ALM13, Theorem 5]) later.

2.1. The comparison principle. Before we state it, we need a little preparation. When we write $\varphi(p, t)$ in (1.1) as graph of function $u(\tilde{x}, t)$, i.e., $\varphi(p, t)=$ $(\tilde{x}(p, t), u(\tilde{x}(p, t), t))$ with unit normal direction $\nu(\tilde{x}, t)=\nu(\varphi(p, t), t)=\frac{1}{\sqrt{|D u|^{2}+1}}(-D u, 1)$ where $D u(\tilde{x}, t)=\left(\partial_{\tilde{x}^{1}} u(\tilde{x}, t), \cdots, \partial_{\tilde{x}^{n}} u(\tilde{x}, t)\right)$, the equation (1.1) becomes

$$
\left\{\begin{array}{l}
\frac{\partial \tilde{x}}{\partial t}=\frac{f\left(\lambda_{t, 1}, \cdots, \lambda_{t, n}\right)}{\sqrt{|D u|^{2}+1}} \cdot D u, \\
\left\langle D u, \frac{\partial \tilde{x}}{\partial t}\right\rangle+\frac{\partial u}{\partial t}=-\frac{f\left(\lambda_{t, 1}, \cdots, \lambda_{t, n}\right)}{\sqrt{|D u|^{2}+1}},
\end{array}\right.
$$

where $\lambda_{t, i}=\lambda_{t, i}(\tilde{x})=\lambda_{t, i}(\tilde{x}(p, t), u(\tilde{x}(p, t), t))$. It follows that the graph function $u(\tilde{x}, t)$ defined on some open subset of $\mathbb{R}^{n} \times[0, T)$ satisfies

$$
\frac{\partial u}{\partial t}=-f\left(\lambda_{t, 1}, \cdots, \lambda_{t, n}\right) \sqrt{|D u|^{2}+1}
$$


Below we will use the following convention for the second fundamental form of graph hypersurface $(\tilde{x}, u(\tilde{x}))$ as used in [An94, $(2.5)], h=\left(h_{A B}\right)=\left(-\frac{D_{A B}^{2} u}{\sqrt{|D u|^{2}+1}}\right)$ where $D_{A B}^{2} u(\tilde{x})=\partial_{\tilde{x}^{A}} \partial_{\tilde{x}^{B}} u(\tilde{x})$.

Proposition 2.1 (B. Andrews). Let $\varphi_{a}: M_{a}^{n} \times[0, T) \rightarrow \mathbb{R}^{n+1}, a=1,2$, be two solutions of Andrews' flow (1.1) with strictly convex initial hypersurfaces $\Sigma_{a, 0}$ where $\Sigma_{a, t}=\varphi_{a}\left(M_{a} \times\{t\}\right) \subset \mathbb{R}^{n+1}$. We further assume that $M_{1}$ is closed, that $M_{2}$ with induced metric by map $\varphi_{2}(\cdot, t)$ is complete for each $t$, that map $\varphi_{2}(\cdot, t): M_{2} \rightarrow \mathbb{R}^{n+1}$ is proper for each $t$, and that the convex hull of $\Sigma_{2,0}$ contains $\Sigma_{1,0}$. Then the distance between hypersurfaces $\Sigma_{1, t}$ and $\Sigma_{2, t}$ is non-decreasing in time $t$.

Proof. Let $\rho(t)$ be the distance between $\Sigma_{1, t}$ and $\Sigma_{2, t}$. Below we will use Hamilton's trick to prove (super-right) derivative $\frac{d}{d t} \rho(t) \geq 0$ whenever $\rho(t)>0$, then the proposition follows.

Fix a $t \in[0, T)$ with $\rho(t)>0$. By the assumption the distance $\rho(t)$ is attained by points $x_{1, t}^{0} \in \Sigma_{1, t}$ and $x_{2, t}^{0} \in \Sigma_{2, t}, \rho(t)=\left|x_{1, t}^{0}-x_{2, t}^{0}\right|$. The tangent planes $T_{x_{1, t}^{0} \Sigma_{1, t}}$ and $T_{x_{2, t}^{0}} \Sigma_{2, t}$ are parallel. Hence $\Sigma_{a, t}$ locally (close to $x_{a, t}^{0}$ ) can be written as graphs over some common small ball in $\mathbb{R}^{n}$. Without loss of generality we may assume that the graph functions are $u_{a}(\tilde{x}, t)$ over open ball $B_{0}^{n}(r)$ of center 0 and radius $r$ such that $x_{a, t}^{0}=\left(0, u_{a}(0, t)\right)$. From the assumption we may also assume that $u_{1}(0, t)<u_{2}(0, t)$, that the normal vectors are $\nu_{a}(\tilde{x}, t)=\frac{1}{\sqrt{\left|D u_{a}\right|^{2}+1}}\left(-D u_{a}, 1\right)$, and that $\Sigma_{a, t} \cap B_{x_{a, t}^{0}}^{n+1}\left(r_{*}\right) \subset$ $\left\{\left(\tilde{x}, u_{a}(\tilde{x}, t)\right), \tilde{x} \in B_{0}^{n}(r)\right\}$ for some $r_{*}>0$ small enough.

By the construction above for the particular $t$ we have $\rho(t)=u_{2}(0, t)-u_{1}(0, t)$ and function $u_{2}(\cdot, t)-u_{1}(\cdot, t)$ on $B_{0}^{n}(r)$ has a positive local minimum at $\tilde{x}=0$. Hence

$$
\left.D\left(u_{2}(\tilde{x}, t)-u_{1}(\tilde{x}, t)\right)\right|_{\tilde{x}=0}=0,\left.\quad D^{2}\left(u_{2}(\tilde{x}, t)-u_{1}(\tilde{x}, t)\right)\right|_{\tilde{x}=0} \geq 0 .
$$

Let $g_{a, t}(\tilde{x})$ and $h_{a, t}(\tilde{x})$ be the induced metric and second fundamental form of hypersurface $\Sigma_{a, t}$ at $\left(\tilde{x}, u_{a}(\tilde{x}, t)\right)$ using the canonical choice of unit normal vectors, respectively. Hence we have that

$$
g_{1, t}(0)=g_{2, t}(0) \quad \text { and } \quad h_{2, t}(0) \leq h_{1, t}(0) .
$$

By [An07, Theorem 1.1] the hypersurfaces $\Sigma_{a, t}$ are strictly convex for each $t$, i.e., the principal curvatures $\lambda_{a, t, i}(\tilde{x})>0$ for each $a, t, i$, and $\tilde{x}$. We compute at the $t$ by using the Hamilton's trick and equations (2.1) and (2.2)

$$
\begin{aligned}
\frac{d}{d t} \rho(t) & =\left.\frac{\partial\left(u_{2}-u_{1}\right)}{\partial t}\right|_{\tilde{x}=0} \\
& =\left.\left(-f\left(\lambda_{2, t, 1}, \cdots, \lambda_{2, t, n}\right) \sqrt{1+\left|D u_{2}\right|^{2}}+f\left(\lambda_{1, t, 1}, \cdots, \lambda_{1, t, n}\right) \sqrt{1+\left|D u_{1}\right|^{2}}\right)\right|_{\tilde{x}=0} \\
& =\left.\left(f\left(\lambda_{1, t, 1}, \cdots, \lambda_{1, t, n}\right)-f\left(\lambda_{2, t, 1}, \cdots, \lambda_{2, t, n}\right)\right) \sqrt{1+\left|D u_{1}\right|^{2}}\right|_{\tilde{x}=0} .
\end{aligned}
$$

Below we use $S$ to denote linear subspace of tangent space $T_{x_{a, t}^{0}} \Sigma_{a, t}$. By Courant minmax principle we have the principal curvature

$$
\lambda_{a, t, i}(0)=\min _{\operatorname{dim} S=i} \max _{y \in S, g_{a, t}(y, y)=1} y h_{a, t}(0) y^{T},
$$


it follows from (2.2) that $0<\lambda_{2, t, i}(0) \leq \lambda_{1, t, i}(0)$ for each $i$. Recall that $f$ is assumed to be strictly monotone increasing in each argument, we conclude that $f\left(\lambda_{2, t, 1}, \cdots, \lambda_{2, t, n}\right) \leq f\left(\lambda_{1, t, 1}, \cdots, \lambda_{1, t, n}\right)$ at $\tilde{x}=0$. We have proved $\frac{d}{d t} \rho(t) \geq 0$.

2.2. Comparing with cylindrical solutions. Since in our construction of ancient solutions we need to compare solutions with cylindrical solutions which are not strictly convex. Here we pay some attention to the difference with Proposition 2.1.

Fixing a $J \in\{1,2, \cdots, n-1\}$, we define the cone $\Gamma_{+, J}^{n}=\left\{\left(\lambda_{1}, \cdots, \lambda_{n}\right) \in \mathbb{R}^{n}, \lambda_{i} \geq\right.$ 0 for $1 \leq i \leq n-J$, and $\lambda_{i}>0$ for $\left.i \geq n-J+1\right\}$. Now we introduce another condition on function $f$.

Assumption $B_{J}$. Given $J \in\{1,2, \cdots, n-1\} f$ can be extended to a continuous function on $\Gamma_{+, J}^{n}$ and $c_{J 0}=f(\underbrace{0, \cdots, 0}_{n-J}, 1, \cdots, 1)>0$.

The principle curvatures of hypersurface $S^{J}(R) \times \mathbb{R}^{n-J}$ are $\lambda_{i}=0$ for $i=1, \cdots, n-$ $J$ and $\lambda_{i}=\frac{1}{R}, i=n-J+1, \cdots, n$. Hence if $f$ satisfies Assumption $B_{J}$, it is easy to check that $S^{J}(R(t)) \times \mathbb{R}^{n-J}$ with $R(t)=\sqrt{(R(0))^{2}-2 c_{J 0} t}$ is a solution of (1.1) where the corresponding map is

$$
\varphi_{J}(y, z, t)=(R(t) y, z) \quad \text { for }(y, z) \in S^{J}(1) \times \mathbb{R}^{n-J} \text { and } t<T_{J},
$$

where $T_{J}=\frac{(R(0))^{2}}{2 c_{J 0}}$ is the singular time of the solutions. Note that the unit normal vector is $\nu(R(t) y, z, t)=(y, 0)$.

Remark 2.2. Fix a $J \in\{1, \cdots, n-1\}$, if we further assume that $f$ in Proposition 2.1 satisfies Assumption $B_{J}$, then the conclusion in the proposition still holds when we take hypersurface $\Sigma_{2, t}$ to be $S^{J}(R(t)) \times \mathbb{R}^{n-J}$. The proof is trivial.

\section{Compactness theorem for Andrews' Flow}

The proof of the following compactness theorem is similar to the proof of An94, Theorem 6.1] (compare to [AMZ13, Theorem 17]).

Theorem 3.1. Let $\varphi_{a}: S^{n} \times\left(\alpha_{a}, 0\right] \rightarrow \mathbb{R}^{n+1}$ be a sequence of solutions to Andrews' flow (1.1) with $\lim _{a \rightarrow \infty} \alpha_{a}=\alpha_{\infty}<0$. Assume that for any $t_{*} \in\left(\alpha_{\infty}, 0\right]$ there is a constant $c_{1}\left(t_{*}\right)<\infty$ such that for index a large enough

$$
\varphi_{a}\left(S^{n} \times\{t\}\right) \subset B_{0}^{n+1}\left(c_{1}\left(t_{*}\right)\right) \quad \text { for all } t \in\left[t_{*}, 0\right] .
$$

We also assume that there is a constant $r_{*}>0$ such that open ball $B_{0}^{n+1}\left(r_{*}\right)$ is contained in the convex hull of $\varphi_{a}\left(S^{n} \times\{0\}\right)$ for all $a$. Then there is a subsequence of $\left\{\varphi_{a}\right\}$ which converges to a strictly convex solution of Andrews' flow $\varphi_{\infty}: S^{n} \times$ $\left(\alpha_{\infty}, 0\right] \rightarrow \mathbb{R}^{n+1}$ in any $C^{\infty}$-topology uniformly on any compact subset of $S^{n} \times\left(\alpha_{\infty}, 0\right]$.

Proof. We define map $\bar{\pi}_{a, t}: S^{n} \rightarrow S^{n}(1)$ by $z=\bar{\pi}_{a, t}(p)=\frac{\varphi_{a}(p, t)}{\left|\varphi_{a}(p, t)\right|}$, clearly the map is one-to-one and onto. We define the radial distance function $r_{a}(\cdot, t): S^{n}(1) \rightarrow(0, \infty)$ by $r_{a}(z, t)=\left|\varphi_{a}\left(\bar{\pi}_{a, t}^{-1}(z), t\right)\right|$. Let $\bar{g}$ and $\bar{\nabla}$ be the Euclidean metric and the Riemannian connection on $S^{n}(1)$, respectively. For a symmetric matrix $B_{n \times n}$ with eigenvalue $b_{1}, \cdots, b_{n}$ we define function $F(B)=f\left(b_{1}, \cdots, b_{n}\right)$. By [An94, Lemma 3.2] we know that $r_{a}(\cdot, \cdot)$ satisfies the following parabolic equation on $S^{n}(1) \times\left(\alpha_{a}, 0\right]$.

$$
\partial_{t} r_{a}(z, t)=-F\left(-\frac{1}{\beta r_{a}(z, t)^{2}} \bar{g}^{*}\left(\bar{\nabla}\left(\beta \bar{\nabla} r_{a}(z, t)\right)\right)+\frac{\mathrm{Id}}{r_{a}(z, t)}\right),
$$


where $\beta=\frac{1}{\sqrt{\left|\bar{\nabla} r_{a}(z, t)\right|^{2}+r_{a}(z, t)^{2}}}$.

Fixed a $t_{*} \in\left(\alpha_{\infty}, 0\right]$, by (3.1) the solution $r_{a}(\cdot, \cdot)$ is uniformly bounded on $S^{n}(1) \times$ $\left[t_{*}, 0\right]$ for all $a$, i.e., the length $r_{a}(z, t) \leq c_{1}\left(t_{*}\right)$. Since under the flow the convex hull $\mathcal{K}_{a, t}$ of $\varphi_{a}\left(S^{n} \times\{t\}\right)$ in $\mathbb{R}^{n+1}$ are decreasing in time $t$ ([An94, p.164, line 4]), by the convexity of the hypersurfaces and the assumption $B_{0}^{n+1}\left(r_{*}\right) \subset \mathcal{K}_{a, 0}$ we conclude that

$$
r_{*} \leq\left\langle\varphi_{a}(p, t), \nu_{a}(p, t)\right\rangle=\frac{r_{a}(z, t)^{2}}{\sqrt{\left|\bar{\nabla} r_{a}(z, t)\right|^{2}+r_{a}(z, t)^{2}}} .
$$

Hence there is a constant $c_{2}=c_{2}\left(t_{*}, r_{*}\right)$ such that $\left|\bar{\nabla} r_{a}(z, t)\right| \leq c_{2}$ on $S^{n}(1) \times\left[t_{*}, 0\right]$ for all $a$. This implies that equation (3.2) is uniformly parabolic on $S^{n}(1) \times\left[t_{*}, 0\right]$.

Because of Assumption A1-A4 the estimates above allow us to apply the EvansKrylov estimate for parabolic equations (see, for example, [Kr87, Theorem 2, p.253], An04 for $n=2)$ to (3.2) and conclude that there is an exponent $\alpha \in(0,1)$ and a constant $c_{3}=c_{3}\left(t_{*}, r_{*}\right)$ such that parabolic norm $\left\|r_{a}\right\|_{C^{2+\alpha, 1+\alpha / 2}\left(S^{n}(1) \times\left[t_{*}, 0\right]\right)} \leq c_{3}$ for all $a$. Note that the uniform upper bound of high order Hölder norms $\left\|r_{a}\right\|_{C^{k+\alpha,(k+\alpha) / 2}\left(S^{n}(1) \times\left[t_{*}, 0\right]\right)}$ for each $k>2$, follows from the standard parabolic Schauder theory. It follows from Arzela-Ascoli theorem that there is a subsequence of $\left\{r_{a}\right\}$ which converges to some $r_{\infty}$ in $C^{\infty}$-topology uniformly on $S^{n}(1) \times\left[t_{*}, 0\right]$.

In the second part of the proof of [An94, Lemma 3.2] Andrews described how to recover maps $\varphi_{a}$ and $\varphi_{\infty}$ (with strictly convex image) from radial length function $r_{a}$ and $r_{\infty}$, respectively. From the discussion it is clear that the subsequence of $\left\{\varphi_{a}\right\}$ converges to $\varphi_{\infty}$ in $C^{\infty}$-topology uniformly on $S^{n} \times\left[t_{*}, 0\right]$ whenever the corresponding subsequence of $\left\{r_{a}\right\}$ converges to $r_{\infty}$ smoothly and uniformly. Because $t_{*}$ is chosen arbitrary in $\left(\alpha_{\infty}, 0\right]$, by a diagonalization argument the theorem is proved.

By running the argument about $r_{a}$ in the proof above only on compact subsets of $S^{n}(1)$, it is easy to see the following compactness theorem with possiblely noncompact limits. Here the base points used for taking the limit are implicitly chosen to be the origin. In the proof we will need the following assumption to assure the uniform ellipticity of the right hand side of partial differential equation (3.2) for the sequence of solutions $r_{a}$ whose corresponding hypersurfaces may have principal curvatures arbitrarily close to 0 .

Assumption E. $\frac{\partial f}{\partial \lambda_{i}}>0$ on the closure $\overline{\Gamma_{+}^{n}} \backslash\{0\}$ for each $i$

Theorem 3.2. Assume $f$ in (1.1) satisfies Assumption E. Let $\varphi_{a}: S^{n} \times\left(\alpha_{a}, 0\right] \rightarrow$ $\mathbb{R}^{n+1}$ be a sequence of solutions to Andrews' flow (1.1) with $\lim _{a \rightarrow \infty} \alpha_{a}=\alpha_{\infty}<0$. Let functions $r_{a}(\cdot, \cdot)$ be defined as in the proof of Theorem 3.1 and let $\Omega \subset S^{n}(1)$ be an open subset. Choose a sequence of smooth compact manifolds with boundary $\Omega_{k} \subset \Omega, k \in \mathbb{N}$, which form an exhaustion of $\Omega$ in the sense that $\Omega_{k} \subset \Omega_{k+1}$ and $\cup_{k} \Omega_{k}=\Omega$. Assume that for any $t_{*} \in\left(\alpha_{\infty}, 0\right]$ and $k$ there is a constant $c_{1}\left(t_{*}, k\right)<\infty$ such that for index a large enough

$$
r_{a}(z, t) \leq c_{1}\left(t_{*}, k\right) \quad \text { for all }(z, t) \in \Omega_{k} \times\left[t_{*}, 0\right] .
$$

We also assume that there is a constant $r_{*}>0$ such that open ball $B_{0}^{n+1}\left(r_{*}\right)$ is contained in the convex hull of $\varphi_{a}\left(S^{n} \times\{0\}\right)$ for all a. Then there is a subsequence of $\left\{\varphi_{a}\right\}$ which converges to a convex solution of Andrews' flow $\varphi_{\infty}: \cup_{t \in\left(\alpha_{\infty}, 0\right]}\left(\tilde{\Omega}_{t}^{n} \times\{t\}\right) \rightarrow$ 
$\mathbb{R}^{n+1}$ in $C^{\infty}$-topology uniformly on any compact subset of $\cup_{t \in\left(\alpha_{\infty}, 0\right]}\left(\tilde{\Omega}_{t} \times\{t\}\right)$. Here domain $\tilde{\Omega}_{t}=\bar{\pi}_{\infty}(\cdot, t)^{-1}(\Omega) \subset S^{n}$ where $\operatorname{map} \bar{\pi}_{\infty}(p, t)=\frac{\varphi_{\infty}(p, t)}{\left|\varphi_{\infty}(p, t)\right|} \in S^{n}(1)$ for $p \in \tilde{\Omega}_{t}$.

\section{Construction of Ancient solutions}

In this section we prove Theorem 1.1 about the existence of ancient solutions and show their forward limits are a round point. At the end we also discuss the noncollapsing property of the solutions. We leave the properties of the backwards limits to the next section.

4.1. Construction of the initial hypersurfaces. Since the existence result in [An07, Theorem 1.1] requires the initial hypersurfaces to be strictly convex, we need to modify the usual construction of initial hypersurfaces (e.g., [HH16, p.597]) so that their principal curvature are positive everywhere.

Fix an integer $J \in\{1,2, \cdots, n-1\}$. For each $a \in \mathbb{N}$ we construct a smooth closed strictly convex hypersurface $\Sigma_{J, a, 0}^{n} \subset \mathbb{R}^{n+1}$ as follows. Let $x=\left(x^{1}, \cdots, x^{n+1}\right)=(y, z)$ be coordinates on $\mathbb{R}^{n+1}$ where $y^{1}=x^{1}, \cdots, y^{J+1}=x^{J+1}$ and $z^{1}=x^{J+2}, \cdots, z^{n-J}=$ $x^{n+1}$. We define $s=\sqrt{\left(z^{1}\right)^{2}+\cdots+\left(z^{n-J}\right)^{2}}$. Fix a constant $\epsilon_{0} \in(0,1)$, we choose a rotationally symmetric function $\psi_{a}$ satisfying

$$
\psi_{a}(z)=\tilde{\psi}_{a}(s)=\left\{\begin{array}{l}
1+\epsilon_{0} \quad \text { if } s=0 \\
1 \quad \text { if } s=a \\
0 \quad \text { if } s=a+1
\end{array}\right.
$$

We further require that $\tilde{\psi}_{a}$ satisfies (i) $\tilde{\psi}_{a}^{\prime}(s)=\frac{d \tilde{\psi}_{a}}{d s}<0$ for $s \in(0, a+1]$, (ii) $\tilde{\psi}_{a}^{\prime \prime}(s)=\frac{d^{2} \tilde{\psi}_{a}}{d s^{2}}<0$ for $s \in[0, a+1]$, (iii) $\tilde{\psi}_{a}(\tilde{s}+a)=\tilde{\psi}_{*}(\tilde{s})$ where $\tilde{s} \in[0,1]$, is a function independent of $a$, and (iv) embedding

$$
\varphi_{J, a, 0}: S^{J}(1) \times B_{0}^{n-J}(a+1) \rightarrow \mathbb{R}^{J+1} \times \mathbb{R}^{n-J}, \quad \varphi_{J, a, 0}(y, z)=\left(\psi_{a}(z) y, z\right)
$$

defines a smooth (including at $|z|=a+1$ ) closed hyperesurface $\sum_{J, a, 0}^{n}$. Intuitively $\Sigma_{J, a, 0}$ is constructed from capping an almost-cylinder $\left\{\left(\psi_{a}(z) y, z\right),(y, z) \in S^{J}(1) \times\right.$ $\left.B_{0}^{n-J}(a)\right\}$ off in a $z$-rotationally-symmetric and strictly convex way (compare with [HH16, p.597]). It is easy to see the existence of such functions $\tilde{\psi}_{a}(s)$.

Now we show that each hypersurface $\Sigma_{J, a, 0}$ is strictly convex by computing its second fundamental form. Because of the $O_{J+1} \times O_{n-J}$-rotational symmetry, we use local coordinate $(\tilde{y}, z)=\left(y^{1}, \cdots, y^{J}, z^{1}, \cdots, z^{n-J}\right) \in B_{0}^{J}(1) \times B_{0}^{n-J}(a+1)$ for $S^{J}(1) \times B_{0}^{n-J}(a+1)$ by taking $y^{J+1}=\sqrt{1-\left(y^{1}\right)^{2}-\cdots-\left(y^{J}\right)^{2}}$. Note that $\left(\tilde{y}, y^{J+1}\right) \in$ $S^{J}(1)$. Let $\left\{e_{\bar{A}}, \bar{A}=1, \cdots, n+1\right\}$ be the standard basis of $\mathbb{R}^{n+1}$. We have the tangent vectors for $\Sigma_{J, a, 0}$ (not necessarily unit)

$$
\begin{aligned}
& \tilde{e}_{i}(\tilde{y}, z)=\frac{\partial}{\partial y^{i}}=\tilde{\psi}_{a}(s) e_{i}-\frac{\tilde{\psi}_{a}(s) y^{i}}{y^{J+1}} e_{J+1}, \quad i=1, \cdots, J \\
& \hat{e}_{p}(\tilde{y}, z)=\frac{\partial}{\partial z^{p}}=\frac{\tilde{\psi}_{a}^{\prime}(s) z^{p}}{s} \sum_{i=1}^{J+1} y^{i} e_{i}+e_{J+1+p}, \quad p=1, \cdots, n-J
\end{aligned}
$$


The unit normal vector of the hypersurface is given by

$$
\nu(\tilde{y}, z)=\frac{1}{\sqrt{1+\left(\tilde{\psi}_{a}^{\prime}(s)\right)^{2}}}\left(\sum_{i=1}^{J+1} y^{i} e_{i}-\frac{\tilde{\psi}_{a}^{\prime}(s)}{s} \sum_{p=1}^{n-J} z^{p} e_{J+1+p}\right) .
$$

Because of the symmetry, below we compute the second fundamental form at point where $\tilde{y}=0$. We have the directional derivatives in $\mathbb{R}^{n+1}$

$$
\begin{aligned}
& D_{j} \tilde{e}_{i}(0, z)=\left.\frac{\partial}{\partial y^{j}} \tilde{e}_{i}(\tilde{y}, z)\right|_{\tilde{y}=0}=-\tilde{\psi}_{a} \delta_{i j} e_{J+1}, \quad j=1, \cdots, J, \\
& D_{q} \tilde{e}_{i}(0, z)=\frac{\tilde{\psi}_{a}^{\prime} z^{q}}{s} e_{i}, \quad q=1, \cdots, n-J, \\
& D_{j} \hat{e}_{p}(0, z)=\frac{\tilde{\psi}_{a}^{\prime} z^{p}}{s} e_{j}, \\
& D_{q} \hat{e}_{p}(0, z)=\left(\frac{\tilde{\psi}_{a}^{\prime \prime} z^{p} z^{q}}{s^{2}}+\frac{\tilde{\psi}_{a}^{\prime}\left(\delta_{p q} s^{2}-z^{p} z^{q}\right)}{s^{3}}\right) e_{J+1},
\end{aligned}
$$

and the unit normal vector

$$
\nu(0, z)=\frac{1}{\sqrt{1+\left(\tilde{\psi}_{a}^{\prime}\right)^{2}}}\left(e_{J+1}-\frac{\tilde{\psi}_{a}^{\prime}}{s} \sum_{p=1}^{n-J} z^{p} e_{J+1+p}\right) .
$$

Hence the second fundamental form $h(0, z)=\left[\begin{array}{cc}-\left\langle D_{j} \tilde{e}_{i}, \nu\right\rangle & -\left\langle D_{q} \tilde{e}_{i}, \nu\right\rangle \\ -\left\langle D_{j} \tilde{e}_{p}, \nu\right\rangle & -\left\langle D_{q} \tilde{e}_{p}, \nu\right\rangle\end{array}\right]$ is given by block matrix

$$
\left[\begin{array}{cc}
\frac{\tilde{\psi}_{a}}{\sqrt{1+\left(\tilde{\psi}_{a}^{\prime}\right)^{2}}} I_{J} & 0 \\
0 & -\frac{1}{s^{3} \sqrt{1+\left(\tilde{\psi}_{a}^{\prime}\right)^{2}}} \Omega_{(n-J) \times(n-J)}
\end{array}\right],
$$

where $I_{J}$ is the identity matrix and matrix $\Omega$ is given by

$$
\left[\begin{array}{cccc}
s \tilde{\psi}_{a}^{\prime \prime}\left(z^{1}\right)^{2}+\tilde{\psi}_{a}^{\prime}\left(s^{2}-\left(z^{1}\right)^{2}\right) & \left(s \tilde{\psi}_{a}^{\prime \prime}-\tilde{\psi}_{a}^{\prime}\right) z^{1} z^{2} & \cdots & \left(s \tilde{\psi}_{a}^{\prime \prime}-\tilde{\psi}_{a}^{\prime}\right) z^{1} z^{n-J} \\
\left(s \tilde{\psi}_{a}^{\prime \prime}-\tilde{\psi}_{a}^{\prime}\right) z^{2} z^{1} & s \tilde{\psi}_{a}^{\prime \prime}\left(z^{2}\right)^{2}+\tilde{\psi}_{a}^{\prime}\left(s^{2}-\left(z^{2}\right)^{2}\right) & \cdots & \left(s \tilde{\psi}_{a}^{\prime \prime}-\tilde{\psi}_{a}^{\prime}\right) z^{2} z^{n-J} \\
\vdots & \vdots & \vdots & \vdots \\
\left(s \tilde{\psi}_{a}^{\prime \prime}-\tilde{\psi}_{a}^{\prime}\right) z^{n-J} z^{1} & \left(s \tilde{\psi}_{a}^{\prime \prime}-\tilde{\psi}_{a}^{\prime}\right) z^{n-J} z^{2} & \cdots & s \tilde{\psi}_{a}^{\prime \prime}\left(z^{n-J}\right)^{2}+\tilde{\psi}_{a}^{\prime}\left(s^{2}-\left(z^{n-J}\right)^{2}\right)
\end{array}\right] .
$$

We can rewrite the matrix above as

$$
\tilde{\psi}_{a}^{\prime}\left[s^{2} I_{n-J}-\left(z^{1}, \cdots, z^{n-J}\right)^{T}\left(z^{1}, \cdots, z^{n-J}\right)\right]+s \tilde{\psi}_{a}^{\prime \prime}\left[\left(z^{1}, \cdots, z^{n-J}\right)^{T}\left(z^{1}, \cdots, z^{n-J}\right)\right] .
$$

It is easy to see that each matrices in the square bracket [ ] above is negative semidefinite and to argue that the matrix in (4.2) is negative definite when $z \neq 0$. It takes a little effort to argue the lower block matrix in (4.1) is positive definite at $z=0$, actually the block matrix equals to $-\tilde{\psi}_{a}^{\prime \prime}(0) I_{n-J}$ at $z=0$.

Thus we can conclude the following.

(C1) Hypersurfaces $\Sigma_{J, a, 0}$ are $O_{J+1} \times O_{n-J}$-rotationally symmetric and strictly convex. (C2) The hypersurfaces $\Sigma_{J, a, 0}$ are uniformly $(n-J+1)$-convex, in the sense that their principal curvatures and mean curvatures satisfy $\lambda_{J, a, 0,1}+\cdots+\lambda_{J, a, 0, n-J+1} \geq \beta H_{J, a, 0}$ 
for some constant $\beta=\beta(n)>0$ independent of $a$. To see this at a point where $\tilde{y}=0$, note that length $\left|\tilde{e}_{i}(0, z)\right|=\tilde{\psi}_{a}(s)$, hence the principal curvatures induced from the eigenvalues in the first block matrix in (4.1) are $\frac{1}{\tilde{\psi}_{a} \sqrt{1+\left(\tilde{\psi}_{a}^{\prime}\right)^{2}}}$ of multiplicity $J$. By the choice of $\tilde{\psi}_{a}$ we know that $\left|\tilde{\psi}_{a}(\tilde{s}+a) \cdot \tilde{\psi}_{a}^{\prime}(\tilde{s}+a)\right| \geq c_{1}$ for all $a$ and $\tilde{s} \in[0,1]$ where $c_{1}>0$ is a constant independent of $a$ and $\tilde{s}$, hence these principal curvatures on $\Sigma_{J, a, 0}$ satisfy $c_{2} \leq \lambda_{J, a, 0, n-J+1} \leq \cdots \leq \lambda_{J, a, 0, n} \leq c_{3}$ for some constant $c_{2}$ and $c_{3}$ independent of $a$. Hence this implies that there is a uniform lower bound for $\lambda_{J, a, 0,1}+\cdots+\lambda_{J, a, 0, n-J+1}$.

Since $\left|\hat{e}_{p}(0, z)\right|=\sqrt{\frac{\left(\tilde{\psi}_{a}^{\prime}(s) z^{p}\right)^{2}}{s^{2}}+1}$, the principal curvatures $\lambda_{J, a, 0, p}, p=1, \cdots, n-J$, are the eigenvalues of matrix (4.2) multiplied by $-\frac{1}{s^{3} \sqrt{1+\left(\tilde{\psi}_{a}^{\prime}\right)^{2}}} \cdot \frac{1}{\left(\tilde{\psi}_{a}^{\prime}\right)^{2} \frac{\left(z^{p}\right)^{2}}{s^{2}}+1}$. Hence these principal curvatures are uniformly bounded from above by some constant $c_{4}$ independent of $a$. This implies that there is a uniform upper bound for the mean curvature $H_{J, a, 0}$. Hence the uniform $(n-J+1)$-convexity is proved.

(C3) The hypersurfaces $\Sigma_{J, a, 0}$ are uniformly non-collapsed from the interior on the scale of $f$ in the sense that there is a constant $\kappa>0$ independent of $a$ such that for every $x \in \Sigma_{J, a, 0}$ there is an interior sphere tangent to $\Sigma_{J, a, 0}$ at $x$ with radius at least $\frac{\kappa}{f\left(\lambda_{J, a, 0,1}(x), \cdots, \lambda_{J, a, 0, n}(x)\right)}$. This is due to the uniform lower bound of function $f\left(\lambda_{J, a, 0,1}(\cdot), \cdots, \lambda_{J, a, 0, n}(\cdot)\right)$ on $\Sigma_{J, a, 0}$, which is a consequence of Assumption $B_{J}$.

4.2. Construction of approximate ancient solutions. Let $\Sigma_{J, a, t}^{n}$ be the Andrews' flow starting from $\Sigma_{J, a, 0}$ constructed in \$4.1 at $t=0$ ([An07, Theorem 1.1]). We know that the flow $\Sigma_{J, a, t}$ collapses to a round point in finite time $T_{J, a}$ and that $\Sigma_{J, a, t}$ is $O_{J+1} \times O_{n-J}$-rotationally symmetric by the uniqueness of the solutions.

We assume that $f$ in the Andrews' flow satisfies Assumption $B_{J}$. Using sphere solution $S^{n}\left(R_{1}(t)\right)$ with $R_{1}(0)=1$ as an interior barrier and cylinder solution $S^{J}\left(R_{2}(t)\right) \times$ $\mathbb{R}^{n-J}$ with $R_{2}(0)=1+2 \epsilon_{0}$ as an exterior barrier, by Proposition 2.1 and Remark 2.2 we see that times $T_{J, a}$ are comparable to one in the sense $\frac{1}{2 f(1, \cdots, 1)} \leq T_{J, a} \leq \frac{\left(1+2 \epsilon_{0}\right)^{2}}{2 c_{J 0}}$. Note that the bounds are independent of $a$.

Let $\hat{\Sigma}_{J, a, \hat{t}}^{n}, \hat{t} \in\left[\hat{T}_{J, a}, 0\right)$ (where $\hat{T}_{J, a}<-1$ denotes the new initial time) be the sequence of solutions of (1.1) obtained by parabolic rescaling of $\Sigma_{J, a, t}$ with $\hat{t}=\Lambda_{J, a}^{2}(t-$ $T_{J, a}$ ) and $\hat{\Sigma}_{J, a, \hat{t}}=\Lambda_{J, a} \Sigma_{J, a, t}$ for some $\Lambda_{J, a}>0$. Define the major radius and the minor radius of hypersurface $\hat{\Sigma}_{J, a, \hat{t}}$ by

$$
A_{J, a}(\hat{t})=\max _{x \in \hat{\Sigma}_{J, a, \hat{t}}}\left(\sum_{i=J+2}^{n+1}\left(x^{i}\right)^{2}\right)^{1 / 2}, \quad \text { and } \quad B_{J, a}(\hat{t})=\max _{x \in \hat{\Sigma}_{J, a, \hat{t}}}\left(\sum_{i=1}^{J+1}\left(x^{i}\right)^{2}\right)^{1 / 2}
$$

respectively. We choose the scaling factor $\Lambda_{J, a}$ such that the ratio $\frac{A_{J, a}(\hat{t})}{B_{J, a}(\hat{t})}$ equals 2 for the first time at $\hat{t}=-1$. In the following we will use the ideas from [HH16, p.598] to prove Claim D1 and D2 stated below.

Claim D1. There exists a constant $C<1$ independent of index $a$ such that diameters

$$
C \leq \operatorname{diam}\left(\hat{\Sigma}_{J, a,-1}\right) \leq C^{-1}
$$


Proof of Claim D1. Since the flows starting from $\hat{\Sigma}_{J, a,-1}$ become extinct in one unit of time, the lower bound of the diameters follows from comparison with a spherical solution as an exterior barrier of the flows. For the upper bound, using the $O_{J+1} \times$ $O_{n-J}$-rotational symmetry, $\frac{A_{J, a}(-1)}{B_{J, a}(-1)}=2$, and the convexity, we can construct a sphere of radius which is a fraction (independent of $a$ ) of the diameters as an interior barrier of $\hat{\Sigma}_{J, a,-1}$. Since the flow becomes extinct in one unit of time, by Proposition [2.1 this barrier sphere solution extincts within one unit of time and so the sphere has a radius less or equal to $\sqrt{2 f(1, \cdots, 1)}$. Hence we have the required upper bound on the diameters.

Now we show that $\left\{\hat{\Sigma}_{J, a, \hat{t}}^{n}\right\}_{a=1}^{\infty}$ is a sequence of approximate ancient solutions of (1.1) by proving

Claim D2. $\lim _{a \rightarrow \infty} \hat{T}_{J, a}=-\infty$.

Proof of Claim D2. Fix a time $\hat{t}_{0}<-1$. Using the $O_{J+1} \times O_{n-J}$-rotational symmetry, $\frac{A_{J, a}\left(\hat{t}_{0}\right)}{B_{J, a}\left(\hat{t}_{0}\right)} \geq 2$, and the convexity, we can put a sphere of radius $\frac{B_{J, a}\left(\hat{t}_{0}\right)}{4}$ inside $\hat{\Sigma}_{J, a, \hat{t}_{0}}$ at distance $\frac{A_{J, a}\left(\hat{t}_{0}\right)}{2}$ away from the origin. The sphere is centered on the plane $\{0\} \times \mathbb{R}^{n-J}$. Thus by Proposition 2.1 it takes function $A_{J, a}(\cdot)$ a time period $\left|\hat{t}_{0}-\hat{t}_{*}\right|$ of at least $\frac{B_{J, a}\left(\hat{t}_{0}\right)^{2}}{32}$ to decrease from $A_{J, a}\left(\hat{t}_{0}\right)$ to $\frac{1}{2} A_{J, a}\left(\hat{t}_{0}\right)=A_{J, a}\left(\hat{t}_{*}\right)$. On the other hand, $B_{J, a}(\hat{t})$ decreases with time and from Claim D1 we know that $B_{J, a}(-1) \geq \delta$ for some $\delta>0$ independent of $a$. Thus, it takes quotient function $\frac{A_{J, a}(\cdot)}{B_{J, a}(\cdot)}$ a time period of at least $\frac{\delta^{2}}{32}$ to decrease from $\frac{A_{J, a}\left(\hat{t}_{0}\right)}{B_{J, a}\left(\hat{t}_{0}\right)}$ to $\frac{1}{2} \frac{A_{J, a}\left(\hat{t}_{0}\right)}{B_{J, a}\left(\hat{t}_{0}\right)}$. Since $\frac{A_{J, a}\left(\hat{T}_{J, a}\right)}{B_{J, a}\left(\hat{T}_{J, a}\right)} \rightarrow \infty$ as $a \rightarrow \infty$ by the construction of initial hypersurface $\Sigma_{J, a, 0}$ and $\frac{A_{J, a}(-1)}{B_{J, a}(-1)}=2$, the claim follows.

4.3. Limiting the approximate ancient solutions. First we verify the assumption after (3.1) for sequence $\left\{\hat{\Sigma}_{J, a, \hat{t}}^{n}\right\}, \hat{t} \in\left[\hat{T}_{J, a},-1\right]$. Recall from the proof at the end of $\S 4.2$ we have $B_{J, a}(\hat{t}) \geq B_{J, a}(-1) \geq \delta$ for all $\hat{t} \leq-1$ where $\delta>0$ is a constant independent of $a$. Since $\hat{\Sigma}_{J, a, \hat{t}}$ is $O_{J+1} \times O_{n-J}$-rotationally symmetric and convex, the existence of a fixed size ball inside $\hat{\Sigma}_{J, a, \hat{t}}^{n}$ follows from $\frac{A_{J, a}(\hat{t})}{B_{J, a}(\hat{t})} \geq 2$.

To see the bound in (3.1), we fix a $\hat{t}_{*}<-1$. We claim that there is a $c_{1}\left(\hat{t}_{*}\right)>0$ such that $B_{J, a}(\hat{t}) \leq c_{1}\left(\hat{t}_{*}\right)$ for all $J, a$, and $\hat{t} \in\left[\hat{t}_{*},-1\right]$. To see the claim by contradiction, we assume that there are sequences $\left\{a_{k}\right\}$ and $\left\{\hat{t}_{k} \in\left[\hat{t}_{*},-1\right]\right\}$ such that $B_{J, a_{k}}\left(\hat{t}_{k}\right) \rightarrow \infty$ as $k \rightarrow \infty$, then the convex hull of $\hat{\Sigma}_{J, a_{k}, \hat{t}_{k}}$ contains ball $B_{0}\left(\rho_{k}\right)$ where radius $\rho_{k} \rightarrow \infty$. Applying Proposition 2.1 to $\hat{\Sigma}_{J, a, \hat{t}}, \hat{t} \in\left[\hat{t}_{k},-1\right]$ and the solution with initial surface $S^{n}\left(\rho_{k}\right)$, we conclude that $\hat{t}_{k} \rightarrow-\infty$ which is a contradiction.

We make another claim that there is a constant $c_{2}\left(\hat{t}_{*}\right)>0$ such that $A_{J, a}(\hat{t}) \leq c_{2}\left(\hat{t}_{*}\right)$ for all $J, a$, and $\hat{t} \in\left[\hat{t}_{*},-1\right]$. To see the claim by contradiction, it follows from $\delta \leq B_{J, a}(\hat{t}) \leq c_{1}\left(\hat{t}_{*}\right)$ that we may assume that there is a sequence of $\left\{a_{k}\right\}$ and $\left\{\hat{t}_{k} \in\left[\hat{t}_{*},-1\right]\right\}$ such that $\frac{A_{J, a_{k}}\left(\hat{t}_{k}\right)}{B_{J, a_{k}}\left(\hat{t}_{k}\right)} \rightarrow \infty$ as $k \rightarrow \infty$, the uniform finite existence time $\left[\hat{t}_{*},-1\right]$ used for $\hat{\Sigma}_{J, a, \hat{t}}$ contradicts with the proof of Claim D2, hence the claim is proved. By the definition of $A_{J, a}(\hat{t})$ and $B_{J, a}(\hat{t})$ we conclude that (3.1) holds for sequence $\left\{\hat{\Sigma}_{J, a, \hat{t}}\right\}, \hat{t} \in\left[\hat{T}_{J, a},-1\right]$. 
Now we may use Theorem 3.1 to conclude that sequence $\hat{\Sigma}_{J, a, \hat{t}}^{n}, \hat{t} \in\left[\hat{T}_{J, a},-1\right]$, subconverges to some strictly convex limit $\hat{\Sigma}_{J, \infty, \hat{t}}^{n}, \hat{t} \in(-\infty,-1]$ in $C^{\infty}$-topology. In the discussion above we may move time -1 closer and closer to 0 , hence by a diagonalization argument we may conclude that we get a limit solution $\hat{\Sigma}_{J, \infty, \hat{t}}, \hat{t} \in(-\infty, 0)$ of (1.1). $\hat{\Sigma}_{J, \infty, \hat{t}}$ becomes a round point at origin as $\hat{t} \rightarrow 0$ ([An07, Theorem 1.1]). The limit is not a round sphere solution because $\frac{A_{J, a}(-1)}{B_{J, a}(-1)}=2$ for $\hat{\Sigma}_{J, \infty, \hat{t}}$.

By the $C^{\infty}$-convergence and the symmetry of $\hat{\Sigma}_{J, a, \hat{t}}$ it is clear that $\hat{\Sigma}_{J, \infty, \hat{t}}$ is $O_{J+1} \times$ $O_{n-J}$-rotationally symmetric. This proves Theorem 1.1 about the existence of the ancient soultions of flow (1.1).

Remark 4.1. (i) Here we assume that $f$ in Theorem 1.1 is concave. From the uniformly non-collapsing property of $\Sigma_{J, a, 0}$ given in (C3) near the end of \$4.1 and the scaling invariance of the non-collapsing property, we may apply [ALM13, Corollary 3] and conclude that the solutions $\hat{\Sigma}_{J, a, \hat{t}}$ are uniformly non-collapsed from the interior on the scale of $f$ for all $a$ and $\hat{t}$. Hence their $C^{\infty}$-limit $\hat{\Sigma}_{J, \infty, \hat{t}}$ are uniformly non-collapsed from the interior on the scale of $f$ for all $\hat{t}$.

(ii) Note that for solution $\hat{\Sigma}_{J, \infty, \hat{t}}$ the ratio of major and minor radius $\frac{A_{J, \infty}(\hat{t})}{B_{J, \infty}(\hat{t})} \rightarrow \infty$ as $\hat{t} \rightarrow-\infty$.

(iii) We define a reflection map $\mathcal{R}: \mathbb{R}^{n+1} \rightarrow \mathbb{R}^{n+1}$ by

$$
\mathcal{R}\left(x^{1}, \cdots, x^{J+1}, x^{J+2}, \cdots, x^{n+1}\right)=\left(x^{1}, \cdots, x^{J+1},-x^{J+2}, \cdots,-x^{n+1}\right) .
$$

It is clear that $\hat{\Sigma}_{J, \infty, \hat{t}}$ is invariant under the reflection, $\mathcal{R}\left(\hat{\Sigma}_{J, \infty, \hat{t}}\right)=\hat{\Sigma}_{J, \infty, \hat{t}}$.

(iv) Note that if we do not care about the properties in (C2) and (C3), the above construction of ancient solutions go through without any change by using $\{(y, z) \in$ $\left.\mathbb{R}^{n+1},|y|^{2}+\frac{|z|^{2}}{a^{2}}=1\right\}$ as the initial hypersurfaces $\Sigma_{J, a, 0}$.

\section{ThE BACKWARD ASYMPtotic Limits OF THE ANCIENT SOLUTIONS}

In this section we consider the backward asymptotic limits of the solutions constructed in Theorem 1.1. In particular, we give a proof of Proposition 1.2. In this section $\hat{\Sigma}_{J, \infty, \hat{t}}^{n}, \quad \hat{t} \in(-\infty, 0)$ denotes the ancient solutions constructed in Theorem 1.1

5.1. Proof of cylinder type backward asymptotical limits. We first define the rescaling of $\hat{\Sigma}_{J, \infty, \hat{t}}^{n}, \hat{t} \in(-\infty, 0)$, which will be used for taking backward asymptotic limits. Since $f$ in (1.1) is homogeneous of degree one, the parabolically-rescaled $\sum_{J, K, t}^{n}=K^{-1} \hat{\Sigma}_{J, \infty, K^{2} t}$ is still a solution of (1.1) for each $K>0$. We consider the limits of this family of solutions when $K \rightarrow \infty$.

Let $A_{J, K}(t)$ and $B_{J, K}(t)$ be the major and minor radius of hypersurface $\Sigma_{J, K, t}$ as defined in (4.3), respectively. From the proof of Theorem 1.1] we have $\frac{A_{J, K}(t)}{B_{J, K}(t)} \geq 2$ for $t \leq-1$ and $K \geq 1$, and $\frac{A_{J, K}(-1)}{B_{J, K}(-1)} \rightarrow \infty$ as $K \rightarrow \infty$.

Fix a $t_{*}<-1$, we claim that there is a positive constant $c_{1}\left(t_{*}\right)$ independent of $K \geq 1$ such that $c_{1}\left(t_{*}\right)<B_{J, K}(t)<c_{1}\left(t_{*}\right)^{-1}$ for all $t \in\left[t_{*},-1\right]$ and $K \geq 1$. First we show that $B_{J, K}(t)$ has a uniform upper bound. Using the $O_{J+1} \times O_{n-J}$-rotational symmetry, 
$\frac{A_{J, K}(t)}{B_{J, K}(t)} \geq 2$, and the convexity, we can construct a sphere of radius $\frac{1}{n} B_{J, K}(t)$ centered at 0 as an interior barrier of $\Sigma_{J, K, t}$. Since the flow starting from $\Sigma_{J, K, t}$ becomes singular within time amount $t_{*}$, from Proposition 2.1 we get that the radius $\frac{1}{n} B_{J, K}(t)$ is bounded from above by a multiple of $\left|t_{*}\right|^{1 / 2}$.

To see that $B_{J, K}(t)$ has a uniform lower bound, using the $O_{J+1} \times O_{n-J}$-rotational symmetry, $\frac{A_{J, K}(t)}{B_{J, K}(t)} \geq 2$, the convexity, and the reflection invariance, we can construct cylinder $S_{0}^{J}\left(B_{J, K}(t)\right) \times \mathbb{R}^{n-J}$ as an exterior barrier of $\Sigma_{J, K, t}$. Since the flow starting from $\Sigma_{J, K, t}$ becomes singular within time amount $t_{*}$, from Remark 2.2 we get a lower bound of the radius $B_{J, K}(t)$ by by a multiple of $\left|t_{*}\right|^{1 / 2}$.

For the family of solutions $\left\{\Sigma_{J, K, t}\right\}$, we choose $\Omega=S^{n}(1) \backslash\left(\{0\} \times S^{n-J-1}(1)\right)$ in Theorem 3.2, it is easy to see that condition (3.3) holds because of the uniform upper bound of $B_{J, K}(t)$ proved above. Assume $f$ in (1.1) satisfies Assumption E, we may apply the theorem to $\left\{\Sigma_{J, K, t}\right\}$ with $t \in(-\infty,-1]$ and get a subsequential limit $\Sigma_{J, \infty, t}^{n}, t \in(-\infty,-1]$. This limit is convex and $O_{J+1} \times O_{n-J}$-rotationally symmetric. It is clear that $\Sigma_{J, \infty, t}$ is invariant under the reflection in (4.5).

To see the limit is a cylinder, fix a $t \in(-\infty,-1]$, we choose a point $\left(0, z_{K, *}\right) \in$ $\Sigma_{J, K, t}$ and let $\ell_{z_{K, *}}$ be a minimal geodesic in $\Sigma_{J, K, t}$ joining $\left(0, z_{K, *}\right)$ and its reflection $\mathcal{R}\left(0, z_{K, *}\right)$. From $\frac{A_{J, K}(t)}{B_{J, K}(t)} \rightarrow \infty$ as $K \rightarrow \infty$, we have $A_{J, K}(t) \rightarrow \infty$, hence the length of geodesic $\ell_{z_{K, *}}$ approaches to infinity as $K \rightarrow \infty$. Since these geodesics all pass through the ball $B_{0}^{n+1}\left(c_{1}^{-1}(t)\right)$ due to the upper bound of $B_{J, K}(t)$, these geodesics sub-limit to a line in $\Sigma_{J, \infty, t}$. Since $\Sigma_{J, \infty, t}$ has nonnegative sectional curvature, by combining the Cheeger and Gromoll splitting theorem and the $O_{J+1} \times O_{n-J}$-rotational symmetry we conclude that $\Sigma_{J, \infty, t}$ splits as $S^{J}\left(r_{*}\right) \times \mathbb{R}^{n-J}$ for some radius $r_{*}>0$. Proposition 1.2 now follows.

5.2. A speculation about the bowl type limits. Choose a sequence of time $\hat{t}_{K} \rightarrow-\infty$ and a sequence of points $\left(0, z_{K}\right) \in \hat{\Sigma}_{J, \infty, \hat{t}_{K}}$, we define dilation scale $Q_{K}$ so that $f\left(\lambda_{K, 1}, \cdots, \lambda_{K, n}\right)=1$ where $\lambda_{K, 1}, \cdots, \lambda_{K, n}$ are the principal curvatures of the dilated and translated hypersurface $Q_{K}\left(\hat{\Sigma}_{J, \infty, \hat{t}_{K}}-\left(0, z_{K}\right)\right)$ at the origin. Based on the knowledge about the ancient solutions of mean curvature flow ([An12], [HH16, Theorem 1.1]) we would like to speculate that the family of solutions $\left\{Q_{K}\left(\hat{\Sigma}_{J, \infty, Q_{K}^{-2} t+\hat{t}_{K}}^{n}-\left(0, z_{K}\right)\right)\right\}$ would sub-converge to a solution of the form Bowl $_{t}^{J} \times \mathbb{R}^{n-J}$ where Bowl $_{t}^{J}$ is a translating soliton solution of Andrews' flow (after dimension reduction) (compare [AW94] and [Wh03], for example). More precisely, let $\lambda_{1}(y), \cdots, \lambda_{J}(y)$ be the principal curvature of Bowl $_{-1}^{J} \subset \mathbb{R}^{J+1}$ at point $y$, then they satisfy

$$
f\left(\lambda_{1}(y), \cdots, \lambda_{J}(y), 0, \cdots, 0\right)=-\left\langle V, \nu_{y}\right\rangle
$$

where $V \in \mathbb{R}^{J+1}$ is a fixed vector and $\nu$ is the unit normal direction. For a special choice of $f$ such translating solitons appear in the work of Brendle and Huisken ([BH15]). Also note that the rotational symmetry of such translating solitons are studied by Bourni and Langford ([BL16]). 


\section{REFERENCES}

[AW94] S. Altschuler and L.F. Wu, Translating surfaces of the non-parametric mean curvature flow with prescribed contact angle. Calc. Var., 2 (1994), 101-111.

[An94] B. Andrews, Contraction of convex hypersurfaces in Euclidean spaces. Calc. Var., 2 (1994), $151-171$.

[An04] B. Andrews, Fully nonlinear parabolic equations in two space variables. arXiv:math.DG/ 0402235v1

[An07] B. Andrews, Pinching estimates and motion of hypersurfaces by curvature functions. J. reine angew. Math. 608 (2007), 17-33.

[ALM13] B. Andrews, M. Langford, and J. McCoy, Non-collapsing in fully non-linear curvature flow. Ann. I. H. Poincare - AN 30 (2013), 23-32.

[AMZ13] B. Andrews, J. McCoy, and Y. Zheng, Contracting convex hypersurfaces by curvature. Calc. Var. Partial Differential Equations 47 (2013), 611-665.

[An12] S. Angenent. Formal asymptotic expansions for symmetric ancient ovals in mean curvature flow. http://www.math.wisc.edu/angenent/preprints.html, 2012.

[ADS18] S. Angenent, P. Daskalopoulos, and N. Sesum. Uniqueness of two-convex closed ancient solutions to the mean curvature flow. arXiv:1804.07230.

[BH15] S. Brendle and G. Huisken, A fully nonlinear flow for two-convex hypersurfaces. arXiv:1507.04651.

[BL16] T. Bourni and M. Langford, Type-II singularities of two-convex immersed mean curvature flow. Geom. Flows, 2 (2016), 1-17.

[BLT17] T. Bourni, M. Langford, and G. Tinglia, Collapsing ancient solutions of mean curvature flow. arXiv: 1705.06981.

[DHS10] P. Daskalopoulos, R. Hamilton, and N. Sesum, Classification of compact ancient solutions to the curve shortening flow. J. Differential Geom., 84 (2010), 455-464.

[DHS12] P. Daskalopoulos, R. Hamilton, N. Sesum, Classification of compact ancient solutions to the Ricci flow on surfaces. J. Differential Geom., 91 (2012), 171-214.

[Ec04] K. Ecker, Regularity Theory for Mean Curvature Flow. Birkhauser, Basel, 2004.

[HH16] R. Haslhofer and O. Hershkovits, Ancient solutions of the mean curvature flow. Comm. Anal. Geom., 24 (2016), 593-604.

[HK16] R. Haslhofer and B. Kleiner, Mean curvature flow of mean convex hypersurfaces. Comm. Pure Appl. Math., 70 (2017), 511-546.

[HIMW] D. Hoffman, T. Ilmanen, F. Martin, and B. White, Graphical translators for mean curvature flow. arViv:1805.10860.

[Kr87] N.V. Krylov, Nonlinear elliptic and parabolic equations of the second order. D. Reidel, 1987.

[Pe02] G. Perelman, The entropy formula for the Ricci flow and its geometric applications. arXiv:math.DG/0211159.

[Wa11] X.J. Wang, Convex solutions to the mean curvature flow. Ann. of Math. (2), 173 (2011), $1185-1239$.

[Wh03] B. White, The nature of singularities in mean curvature flow of mean convex sets. J. Amer. Math. Soc., 16 (2003), 123-138.

Department of Mathematics, University of Oregon, Eugene, OR 97403

E-mail address: penglu@uoregon.edu

School of Math. Science, Yangzhou University, Yangzhou, Jiangsu 225002, China

E-mail address: zhoujiuru@yzu.edu.cn 